\title{
A Plane of Weakly Coupled Heisenberg Chains: Theoretical Arguments and Numerical Calculations
}

\author{
Ian Affleck \\ Canadian Institute for Advanced Research and Physics Department, University of British \\ Columbia, \\ Vancouver, British Columbia, Canada V6T $1 Z 1$ \\ Martin P. Gelfand \\ Department of Physics, Colorado State University, Fort Collins, Colorado 80523 \\ Rajiv R. P. Singh \\ Department of Physics, University of California, Davis, California 95616
}

\begin{abstract}
The $S=1 / 2$, nearest-neighbor, quantum Heisenberg antiferromagnet on the square lattice with spatially anisotropic couplings is reconsidered, with particular attention to the following question: at $T=0$, does Néel order develop at infinitesimal interchain coupling, or is there a nonzero critical coupling? A heuristic renormalization group argument is presented which suggests that previous theoretical answers to that question are incorrect or at least incomplete, and that the answer is not universal but rather depends on the microscopic details of the model under consideration. Numerical investigations of the nearest-neighbor model are carried out via zero-temperature series expansions about Ising and dimer Hamiltonians. The results are entirely consistent with a vanishing critical interchain coupling ratio $R_{c}$; if $R_{c}$ is finite, it is unlikely to substantially exceed 0.02 .
\end{abstract}


PACS: 75.10.-b, 75.10.Jm

Short title: A Plane of Weakly Coupled Heisenberg Chains 


\section{INTRODUCTION}

Lately there has been considerable interest in one-dimensional to two-dimensional crossover in Luttinger liquids. The motivation is largely the interest in possible non-Fermi liquid behavior in two dimensional models for high- $T_{c}$ superconductors [1]. While much of this work has focused on the Hubbard or $t-J$ model, a simpler case to understand is that of the spatially anisotropic square-lattice spin-1/2 Heisenberg antiferromagnet,

$$
H=\sum_{\mathbf{r}_{i}-\mathbf{r}_{j}=e_{x}} J_{x} \mathbf{S}_{i} \cdot \mathbf{S}_{j}+\sum_{\mathbf{r}_{i}-\mathbf{r}_{j}=e_{y}} J_{y} \mathbf{S}_{i} \cdot \mathbf{S}_{j},
$$

(and see Fig. 囵) with $0<J_{y} \ll J_{x}$. The essential question is whether Néel order sets in for infinitesimal

$$
R \equiv J_{y} / J_{x}
$$

or whether there is a finite minimum coupling ratio necessary for long range order (LRO). A subsidiary question is the nature of the magnetically disordered phase at small $R$, if the latter scenario holds true. While various analytical and numerical investigations of this question have appeared in the literature [2 [4, it appears to be far from settled.

The purpose of this paper is twofold. In Sec. [T we review previous analytic arguments for or against the existence of a finite minimum ratio. We then discuss a renormalization group framework within which previous arguments appear to be incorrect or incomplete. This approach indicates that the behavior is not universal and that for any particular model the question may only be answered by numerical investigation. Therefore in Sec. III we present such a numerical investigation of the Hamiltonian (1.1) based on high order series expansion about both the Néel and dimer phases. That analysis seems to indicate that if there is a critical ratio it is rather small.

\section{THEORETICAL ARGUMENTS}




\section{A. Spin-Wave Theory}

Spin-wave theory plays an important role in our understanding of antiferromagnetism. A systematic $1 / S$ expansion (where $S$ is the spin magnitude) is generally believed to give, in low orders, a fairly accurate estimate of the sublattice magnetization on various lattices. In particular, it correctly predicts the absence of long range order at $T=0$ for a onedimensional antiferromagnet. This effect can be seen from the leading order correction to the sublattice magnetization. For the anisotropic 2D model of Eq. (1.1), we have:

$$
\left\langle S_{i}^{z}\right\rangle= \pm\left[S-\frac{1}{2}\left(\int \frac{d^{2} \mathbf{k}}{(2 \pi)^{2}} \frac{1}{\sqrt{1-\gamma_{\mathbf{k}}^{2}}}-1\right)\right]
$$

where

$$
\gamma_{\mathbf{k}} \equiv \frac{J_{x} \cos k_{x}+J_{y} \cos k_{y}}{J_{x}+J_{y}}
$$

and the $\mathbf{k}$ integral runs over the Brillouin zone, $\left|k_{i}\right|<\pi$. For $J_{y}=0$, the integrand is independent of $k_{y}$ and the integral has logarithmic divergences at $k_{x}=0$ and $\pi$ indicating the absence of Néel order. For finite $J_{y}$ the integral is finite and for small $R$ we obtain:

$$
\left\langle S_{i}^{z}\right\rangle \approx \pm\left[S+\frac{1}{\pi} \ln R\right]
$$

If we took as a criterion for the stability of the Néel phase that the $O(1)$ correction should be smaller than the leading $O(S)$ term, then we would conclude that Néel order breaks down at:

$$
R_{c} \approx e^{-\pi S}
$$

For $S=1 / 2$, numerical evaluation of the integral in Eq. (2.1) gives $R_{c} \approx .03367$ [2].

\section{B. Chain Mean Field Theory}

A standard method for treating dimensional crossover problems of this type is to use the known behavior of the antiferromagnetic susceptibility for the one-dimensional system and 
to treat the couplings in the second dimension in mean field theory [5]. (We refer to this as "chain" mean field theory to distinguish it from another mean field theory to be discussed later.) The Hamiltonian of Eq. (1.1) is replaced by the following mean field Hamiltonian for each chain:

$$
H_{C M F} \equiv \sum_{i} J_{x} \mathbf{S}_{i} \cdot \mathbf{S}_{i+1}+2 J_{y}\left\langle S^{z}\right\rangle \sum_{i}(-1)^{i} S_{i}^{z}
$$

We now calculate $\left\langle S^{z}\right\rangle$ using this Hamiltonian and demand self-consistency. The mean field critical point is then determined by:

$$
1=2 R \chi_{1}\left(T_{N}\right)
$$

where $\chi_{1}$ is the zero-frequency antiferromagnetic susceptibility for the one-dimensional chain with $J_{x}=1$. For a half-integer spin Heisenberg antiferromagnet, $\chi_{1}(T)$ diverges as $1 / T$, as $T \rightarrow 0$. This argument then predicts a finite Néel temperature:

$$
T_{N} \approx J_{y}
$$

No matter how small $J_{y}$, the ground state is always ordered. On the other hand, for integer $S, \chi_{1}(0)$ has a finite value of order $1 / \Delta$, the Haldane gap. Hence this argument predicts a disordered ground state for integer $S$ and $R<R_{c}$ with $R_{c} \approx \Delta$. This result is readily generalized to $d$-dimensional systems. The factor of 2 in Eq. (2.6) is simply replaced by the number of nearest neighbor chains. Indeed, it presumably becomes exact in the limit $d \rightarrow \infty$. It has an obvious problem for $d=2$ where the Mermin-Wagner theorem tells us that Néel order should be impossible at any finite temperature. Nonetheless, we might be tempted to believe the conclusion that it does occur at $T=0$. A more sensible application of this mean field theory for $d=2$ is to the case of a staggered intra-chain coupling at $T=0$ (see Sec. ПIIB, below). Eq. (2.6) then becomes:

$$
1=2 R \lambda_{\mathrm{CMF}}^{c} \chi_{1}\left(\lambda_{\mathrm{CMF}}^{c}\right)
$$

Here $\lambda$ is the ratio of coupling on alternate links, and $\lambda_{\mathrm{CMF}}^{c}$ is its critical value within the chain mean field theory. Since a staggered interaction in one dimension has scaling dimension 
$1 / 2$, we conclude that $\chi_{1}(\lambda) \propto(1-\lambda)^{-2 / 3}$ (up to log corrections). Now $1-\lambda$ plays a role roughly analogous to a finite temperature; as $\lambda \rightarrow 1$, the mean field theory predicts that Néel order sets in at $1-\lambda_{\mathrm{CMF}}^{c} \propto R^{3 / 2}$.

\section{Renormalization Group Argument}

Consider a system of quantum chains weakly coupled to each other at $T=0$. They may be of $X Y$ or Heisenberg symmetry. Alternatively, consider a system of classical spins consisting of planes which are weakly coupled to each other, at finite $T$. In either case (ignoring topological terms, for the moment), we may represent the system at long length scales by a 3 -dimensional non-linear $\sigma$-model, with action or Hamiltonian:

$$
S=(\Lambda / 2 g) \int d^{3} \mathbf{x}\left[\left(\partial_{x} \vec{\phi}\right)^{2}+\left(\partial_{y} \vec{\phi}\right)^{2}+R\left(\partial_{z} \vec{\phi}\right)^{2}\right]
$$

Here $\vec{\phi}$ has unit length and either 2 or 3 components in the $X Y$ or Heisenberg case. As before, $R$ is the ratio of inter-chain (or plane) coupling to in-chain (or plane) coupling. $\Lambda$ is the ultraviolet cutoff; i.e., the field, $\vec{\phi}$ has Fourier modes with $\left|k_{i}\right|<\Lambda$. (We cut off the momentum inside a cube.) The factor of $\Lambda$ is inserted to make the three-dimensional coupling constant $g$ dimensionless, as is usually done in formulating the 3D renormalization group (RG) equations.

Note that the only trace of the quasi-2-dimensionality is the anisotropy in the $(\partial \vec{\phi})^{2}$ terms. We can get rid of this by a rescaling of $z$ by a factor of $\sqrt{R}$, that is, we define a new length co-ordinate, $z^{\prime} \equiv z / \sqrt{R}$. In momentum space, we define a new 3-component of momentum, $k_{3}^{\prime} \equiv \sqrt{R} k_{3}$. The action now looks completely 3-dimensional except that the cut-off is no longer a 3D cube but a squat box of area $\Lambda^{2}$ and height $\sqrt{R} \Lambda$. To complete the elimination of the anisotropy from the action, we reduce the length and width of the box to $\sqrt{R} \Lambda$ also, by integrating out higher momentum modes, the standard RG procedure. Because the inter-chain coupling is so weak, this may essentially be done using the $2 \mathrm{D}$ RG equations; in momentum space, $\left(k_{z}^{\prime}\right)^{2}$ is so small (due to the small cut-off) that we 
consider the planes to be essentially decoupled until we have lowered the 2D cutoff down to approximately $\sqrt{R} \Lambda$. The value of the $2 \mathrm{D}$ effective coupling, when the cut-off has been reduced to $\sqrt{R} \Lambda$, then acts as the initial condition for further RG calculations: as we lower the cut-off still further we should use the isotropic 3D RG equations using $g_{2}(\sqrt{R} \Lambda)$ as the initial value. In the limit $R \rightarrow 0$, the $2 \mathrm{D}$ coupling flows to its zero-cutoff fixed point, $g_{2}(0)$. See Fig. 2 for a sketch of one possible flow diagram.

In both $X Y$ and Heisenberg cases, the 3D system has ordered and disordered phases, separated by some critical coupling $g_{c}$. (For $g<g_{c}$ the system is ordered.) Thus, whether or not the system orders for arbitrarily small $R$ is determined by whether or not $g_{2}(0)<g_{c}$. In the case of Heisenberg symmetry with no topological term, $g_{2}(0)$ is naively infinite, so the 3D system is in the disordered phase for sufficiently small $R$. From another point of view, the $2 \mathrm{D}$ system develops a finite correlation length, $\xi$, as we reduce the cut-off. For sufficiently small $R, \sqrt{R} \Lambda<1 / \xi$. Then further renormalization using the $3 \mathrm{D}$ RG equations cannot eliminate this finite correlation length. In the $X Y$ case $g_{2}(0)$ is basically the renormalized dimensionless temperature. It has a finite value along the Kosterlitz-Thouless (KT) critical line, $g_{2}(0)<g_{K T}$. The important question is whether or not $g_{K T}<g_{c}$. If it is, then an arbitrarily weak inter-plane coupling leads to order for all $T<T_{K T}$. In the other case, if $g_{c}<g_{K T}$, then there will be some special temperature, $T_{c}<T_{K T}$, such that below this temperature an arbitrarily weak inter-plane coupling leads to LRO but above this temperature there is a minimum inter-plane coupling necessary for LRO.

Numerical simulations of the classical layered $X Y$ model indicate that $g_{K T}<g_{c}$ [6]. In this case the present approach predicts a critical coupling, $g_{c}(R)$, for weakly coupled planes, slightly above $g_{K T}$. This can be estimated as the bare coupling for which the renormalized coupling at scale $\sqrt{R} \Lambda, g_{2}(\sqrt{R} \Lambda) \approx g_{c}$. Using the 2D Kosterlitz renormalization group equations this gives:

$$
g_{c}(R)-g_{K T} \propto 1 /(\ln R)^{2}
$$

in agreement with estimates based on plane mean field theory [6] and another approach [7,8] 
somewhat closer in spirit to the present one.

Now let us consider the half-integer spin quantum Heisenberg chain [9]. The twodimensional $\sigma$-model action now has an extra topological term added to it, with topological angle $\pi$. This angle itself does not renormalize, for symmetry reasons, but it has a crucial effect on the renormalization of $g_{2}$. There is now a finite coupling critical point, $g_{2}(0)$, shown in Fig. 2. (The strong coupling phase is spontaneously dimerized; it can be reached with a sufficiently strong antiferromagnetic second nearest neighbor coupling.) The threedimensional RG flows are expected to be the same for both integer and half-integer spin, although the nature of the disordered phase may depend on whether the spin is half-integer, odd integer or even integer, due to a Berry's phase topological term [10]. We assume the only important effect of the topological terms is to produce a finite $g_{2}(0)$ critical point in two dimensions, as shown in Fig. 2. The situation is then similar to the classical $X Y$ case. Whether or not a disordered phase occurs for weak interchain coupling depends on whether or not $g_{2}(0)>g_{c}$.

Let us contrast this approach to either of those discussed above, in Secs. ПIA and ПВ. First consider the calculation of the breakdown of the Néel state in lowest order spin-wave theory. This can be regarded as the above calculation of the renormalization of the 2D coupling, using only the lowest order $2 D R G$ equations. For large $S$ we begin with a very small bare coupling. However, the lowest order RG equations always break down for small enough $R$. Furthermore, these equations do not distinguish integer and half-integer $S$. In $\sigma$-model language, the lowest order RG is independent of the topological term. Actually the RG equations are independent of it to all orders in $g$; its effects are exponentially small in $g$. Nonetheless, for small enough $R$ we always renormalize into the regime where these nonperturbative contributions are important. Blind use of the lowest order equations essentially leads to the conclusion that $g_{2}(0)$ is infinite and hence that the system is disordered at small enough $R$.

On the other hand consider the standard mean field argument [5]. Because the 2D susceptibility is divergent along the whole KT critical line, or in the Heisenberg case with 
topological term, it predicts LRO in all these cases. We argue that in order to go beyond a mean field treatment one should consider not the $2 \mathrm{D}$ susceptibility but rather the renormalized 2D effective coupling. Naively if the 2D susceptibility is infinite, the $2 \mathrm{D}$ effective coupling is zero. But in fact, this is not actually the case. Since the 2D effective coupling is finite at zero cut-off we must consider whether or not it is below $g_{c}$, leading to the above conclusion.

It was suggested recently by Parola et al. [4] that, for $R$ less than a finite $R_{c}$, the long wavelength behavior "can be interpreted in terms of decoupled one dimensional chains." The implausibility of this proposal can be seen by considering a finite number of chains. In this case, we can analyze the scaling behavior entirely in terms of the $(1+1)$-dimensional renormalization group. The inter-chain coupling, of dimension 1, is relevant. We might then naively expect from standard scaling arguments that for two chains there should be a gap, proportional to $R$ (up to logarithms). The existence of a gap in this case has been shown numerically [11,12]. In the case of three chains, White et al. [12 found that the gap appears to vanish and this has been argued to be the case for any odd number of chains. However, this does not necessarily mean that the system asymptotically behaves like decoupled chains. Indeed, it seems more likely that the low energy states would be those of a single $S=1 / 2$ chain. This assertion is motivated by the behavior of a single spin $S$ chain as a function of $S$. For integer $S$ one expects a gap while for half-integer $S$ one expects universal gapless behavior which is independent of $S$ (i.e., always in the free boson or $k=1$ Wess-ZuminoWitten universality class). For half-integer $S$ the density of low-lying excitations (after scaling out the spin-wave velocity) does not increase with increasing $S$. We may expect similar behavior for $2 S$ coupled spin-1/2 chains: inter-chain coupling is relevant and the system scales away from decoupled chains to the single chain fixed point with fewer gapless degrees of freedom. Note that in this case, the number of low-lying degrees of freedom does not scale with the area of the system but only with the length. It is of course possible that, as the number of chains is increased, the gap to other excitations decreases and asymptotically approaches zero as the number of chains goes to $\infty$. Indeed, this must happen if the $\infty$-chain 
system Néel orders. However, it then seems unlikely that the low-lying excitations would be those of decoupled chains. We rather expect that, if a disordered phase exists at small $R$, it has a genuinely 2-dimensional nature. Previous work on the isotropic $S=1 / 2$ square lattice Heisenberg antiferromagnet with next-nearest neighbor interactions suggests that a dimer-ordered ground state is the most likely to occur in a magnetically disordered phase 13. However, other possibilities with or without a gap cannot be ruled out.

Actually, the result of our reasoning is pretty uninformative. It is simply that we cannot tell from RG arguments whether or not the system orders for arbitrarily weak inter-plane coupling, in cases where the 2D system has an infinite correlation length. We might expect that the existence or non-existence of a critical $R$ is not universal. Different realizations of the model may have different values of $g_{c}$ or $g_{2}(0)$, since critical temperatures (or couplings) are in general not universal. For instance, a different phase diagram might ensue if the chains are coupled with a first and second nearest neighbor inter-chain coupling, both proportional to $R$. It would thus seem that the question of the existence of a disordered phase for finite $R$ in a particular model must be answered by numerical work.

\section{SERIES CALCULATIONS AND ANALYSES}

We now turn to numerical studies of weakly coupled, $S=1 / 2$ Heisenberg chains, and in particular the anisotropic two-dimensional lattice of Fig. 1 and Eq. (1.1). It is obviously not possible for any numerical calculation to distinguish between $J_{y}^{c}=0$, i.e., the persistence of Néel order for arbitrarily small interchain couplings, and a sufficiently small but positive $J_{y}^{c}$. Provided that there is no strong evidence for any particular nonzero value of $J_{y}^{c}$, the best one can do is argue that numerical data are consistent with a vanishing $J_{y}^{c}$ and offer reasonable upper bounds on its value.

Our numerical studies consist of a variety of zero-temperature series expansions (i.e., Rayleigh-Schrödinger perturbation theory) following the cluster-expansion techniques described in Ref. 14. Series expansions have several advantages over finite-size calculations in 
two-dimensional lattice quantum many-body problems. There is no need to worry about cluster-shape effects — which should be of particular concern in anisotropic models such as the one of present interest. Given comparable computing power, series calculations can account for much further-range correlations than would be possible in an exact diagonalization calculation (see the discussion in Sec. V of Ref. 14). Finally, the fact that the series calculations do not directly study the model of interest, but rather yields results on a one (or more) parameter family of models, may allow for further informative comparisons with approximate analytic calculations.

Expansions were carried out about both Ising and dimer Hamiltonians. The calculations and analyses will be described in detail below; here let us preview the results. The results

of both types of expansions are consistent with $J_{y}^{c}=0$. We believe that $J_{y}^{c}$ is unlikely to exceed $0.02 J_{x}$. This upper bound is not much smaller than the lowest-order spin-wave estimate (and hence by itself cannot be taken as very strong evidence that spin-wave theory is qualitatively wrong); however it is notably less than the value $0.1 J_{x}$ suggested by Parola et al. [4] based on exact diagonalization of clusters with up to 32 spins. In addition, the Ising expansions yield estimates for the staggered magnetization and the correlation-length anisotropy which are in excellent agreement with spin-wave theory for $J_{y} / J_{x}$ down to 0.1 . Finally, the dimer expansions appear to be consistent with the chain mean field theory of Sec. IIB at small $J_{y}$, and thus support the proposition that $J_{y}^{c}=0$. However, that conclusion must be tempered by comparison of the dimer expansions for a plane of chains with the corresponding calculations for a pair of chains.

\section{A. Ising expansions}

In order to discuss the Ising expansions, we consider a generalization of the coupled-chain Hamiltonian (1.1) in which Ising anisotropy is introduced, namely

$$
H=\sum_{\mathbf{r}_{i}-\mathbf{r}_{j}=e_{x}} J_{x}\left[S_{i}^{z} S_{j}^{z}+\alpha\left(S_{i}^{x} S_{j}^{x}+S_{i}^{y} S_{j}^{y}\right)\right]
$$




$$
+\sum_{\mathbf{r}_{i}-\mathbf{r}_{j}=e_{y}} J_{y}\left[S_{i}^{z} S_{j}^{z}+\alpha\left(S_{i}^{x} S_{j}^{x}+S_{i}^{y} S_{j}^{y}\right)\right]
$$

Henceforth we choose units of energy so that $J_{x}=1$ (and $J_{y}=R$ ). Physical quantities are expanded in powers of $\alpha$; we have calculated the ground state energy $E_{g}$, the sublattice magnetization $M=\left\langle S_{0}^{z}\right\rangle$, and the correlation length anisotropy $\left(\xi_{y} / \xi_{x}\right)^{2}$ which is given explicitly by

$$
\sum_{i}\left\langle S_{0}^{z} S_{i}^{z}\right\rangle_{c} y_{i}^{2} / \sum_{i}\left\langle S_{0}^{z} S_{i}^{z}\right\rangle_{c} x_{i}^{2}
$$

where the subscript $c$ refers to connected correlations

$$
\left\langle S_{0}^{z} S_{i}^{z}\right\rangle_{c}=\left\langle S_{0}^{z} S_{i}^{z}\right\rangle-\left\langle S_{0}^{z}\right\rangle\left\langle S_{i}^{z}\right\rangle
$$

The energy and magnetization series were determined to order $\left(\alpha^{2}\right)^{5}$, while the $\left(\xi_{y} / \xi_{x}\right)^{2}$ series were only calculated to order $\left(\alpha^{2}\right)^{4}$. Note that each value of $J_{y}$ requires a separate calculation of the series; since it is not possible to present the complete series expansions in a compact format they are not displayed here, but are available as supplementary material. Here we discuss the analysis.

The energy series were analyzed by direct Padé approximants. The results of five different Padé approximants evaluated at $\alpha=1$ are shown in Fig. 3. It is clear that they extrapolate smoothly between the one and two dimensional limits, that is to say between $J_{y}=0$ and $J_{y}=1$. This is consistent with the expansion being convergent up to $\alpha=1$ for all $J_{y} \geq 0$, which is in turn consistent with $J_{y}^{c}=0$. However, this is quite weak evidence, as we will discuss in connection with the dimer expansions. Perhaps the results for $E_{g}$ could serve best as a touchstone for the quality of finite-size calculations.

To analyze the magnetization series, we first make a change of variables originally introduced by Huse, $\delta=1-\left(1-\alpha^{2}\right)^{1 / 2}$, which removes the square-root singularity at $\alpha=1$ expected on the grounds of spin-wave theory. The results for five Padé approximants evaluated at $\alpha=1$ are shown in Fig. $甘$. For $J_{y} \geq 0.2$ the approximants are well-converged and appear to be in good agreement with spin-wave theory. For $J_{y}<0.2$ the convergence is poor, but the approximants are suggestive of Néel order for all $J_{y}$ and with $M$ vanishing as 
a small power of $J_{y}$. Note that the chain mean field theory implies $M \sim J_{y}^{1 / 2}$ as $J_{y} \rightarrow 0$. In any case, these data provide no evidence that $M \rightarrow 0$ at any particular $J_{y}^{c}>0$.

The correlation length anisotropy series are short, however this quantity is nonsingular as $\alpha \rightarrow 1$ and the Padé approximants evaluated at $\alpha=1$ are extremely consistent even down to very small $J_{y}$ : see Fig. 5. For $J_{y} \geq 0.1$ there is remarkable agreement between the series estimates and the spin-wave calculation presented by Parola et al. [4] For smaller $J_{y}$, two of the three approximants indicate $\xi_{y} / \xi_{x} \rightarrow 0$ for $J_{y}$ between 0 and 0.02 , and the other approximant is ill-behaved. These data are consistent with $J_{y}^{c}=0$, but also with a small critical interchain coupling.

\section{B. Dimer expansions}

The dimer expansions are in the variable $\lambda$ for properties of the Hamiltonian $H=$ $H_{0}+\lambda H_{1}$, where for $H_{0}$ we take the columnar dimer Hamiltonian

$$
H_{0}=\sum_{\langle i j\rangle \in \mathcal{D}} J_{x} \mathbf{S}_{i} \cdot \mathbf{S}_{j}
$$

with $\mathcal{D}$ the dimer covering of the square lattice shown in Fig. 6, and $H_{1}$ is the remainder of the couplings, so that $\lambda=1$ corresponds to the coupled-chain model of interest, namely

$$
H_{1}=\sum_{\mathbf{r}_{i}-\mathbf{r}_{j}=e_{x} ;\langle i j\rangle \notin \mathcal{D}} J_{x} \mathbf{S}_{i} \cdot \mathbf{S}_{j}+\sum_{\mathbf{r}_{i}-\mathbf{r}_{j}=e_{y}} J_{y} \mathbf{S}_{i} \cdot \mathbf{S}_{j}
$$

The quantities for which we have obtained series expansions, to order $\lambda^{7}$ (which involves evaluation of 1041 graphs), include the ground state energy $E_{g}$, moments of the antiferromagnetic equal-time structure factor

$$
\begin{gathered}
M_{1}=\sum_{i}\left\langle\mathbf{S}_{0} \cdot \mathbf{S}_{i}\right\rangle \pi\left(\mathbf{r}_{i}\right), \\
M_{r r}=\sum_{i}\left\langle\mathbf{S}_{0} \cdot \mathbf{S}_{i}\right\rangle \pi\left(\mathbf{r}_{i}\right) r_{i}^{2},
\end{gathered}
$$

(where $\pi\left(\mathbf{r}_{i}\right)=1$ if $\mathbf{r}_{i}$ lies on the same sublattice as $\mathbf{r}_{0}$, and is -1 otherwise), and the antiferromagnetic susceptibility $\chi$. 
It should be clear from the form of the Hamiltonian that all these quantities possess two-variable expansions, in powers of $\lambda$ and $\lambda J_{y}$. Hence the coefficient of $\lambda^{m}$ in any of these series can be expresses in terms of an order- $m$ polynomial in $J_{y}$; and calculations of the seventh-order series at eight values of $J_{y}$ allows one to determine them at any $J_{y}$ by means of polynomial interpolation.

Before considering the details of the dimer series analysis, let us look at the notions underlying this approach, and what we might expect to learn from it. Properties of the Néelordered phase are inaccessible to the dimer expansions (in contrast to the Ising expansions); what they can provide are estimates of $\lambda^{c}\left(J_{y}\right)$, the smallest value of $\lambda$ for a given $J_{y}$ at which the antiferromagnetic correlation length and susceptibility diverge. If one assumes the simplest possible phase diagrams in the $J_{y^{-}} \lambda$ plane, sketched in Fig. 7, then $\lambda^{c}\left(J_{y}\right)<1$ implies that the uniformly coupled system $(\lambda=1)$ exhibits Néel order for that value of $J_{y}$. Furthermore, one may compare the series estimates of $\lambda^{c}\left(J_{y}\right)$ with approximate analytic calculations for the critical line.

Let us first briefly discuss the ground state energy series. Direct Padé approximants allow for estimates of $E_{g}\left(J_{y}, \lambda=1\right)$ which are shown in Fig. 3. The approximants are consistent with each other - and with the values obtained from the Ising expansions for $J_{y}$ as large as 0.4. This implies that the singularity in $E_{g}$ at the critical line is very weak; the alternative, that $\lambda^{c}>1$ for $J_{y}<0.4$, is ruled out by both the Ising expansions and the other dimer expansions.

The terms of the $M_{1}, M_{r r}$, and $\chi$ series are all positive and increasing with order in $\lambda$. Thus one may estimate $\lambda^{c}\left(J_{y}\right)$ either by ratio analyses or inhomogeneous differential approximants (of which Dlog-Padé approximants are a special case) [15].

The estimates of $\lambda^{c}\left(J_{y}\right)$ which result from consideration of $M_{1}$ are poorly converged, so we will only discuss $M_{r r}$ and $\chi$. The results for these two sets of series are presented in Fig. 8. Let us address what the various curves and points signify. First, six differential approximant estimates for $\lambda^{c}\left(J_{y}\right)$ are presented for several values of $J_{y}$; these particular approximants are chosen because they utilize all the terms in the series and they yield good 
estimates of $\lambda^{c}$ at both $J_{y}=0$ and $J_{y}=1$. The missing approximants at any $J_{y}$ are either defective or (in one or two cases) off-scale. The approximants are reasonably consistent over the entire range of $J_{y}$. Second, the thin solid and long-dashed lines are the estimates of $\lambda^{c}$ based on ratio analysis of the three highest-order terms in the $\chi$ and $M_{r r}$ series. For a series $\sum_{n} c_{n} \lambda_{n}$, the ratios $c_{n} / c_{n-1}$ are plotted versus $1 / n$ and pairwise linearly extrapolated to $1 / n=0$; the intercepts yield estimates of $1 / \lambda^{c}$ based on three consecutive terms of the series. The $\chi$ ratio curve lies close to the differential approximants, which reflects the fact that the $\chi$ series are extremely well-behaved. The ratio plot (corresponding to $J_{y}=1$ ) in Fig. 2 of Ref. 14 provides further evidence that the antiferromagnetic susceptibility series is better behaved than other dimer expansions. Both the ratio and differential approximant analyses suggest that $\lambda^{c}\left(J_{y}\right)<1$ for all $J_{y}>0$, and hence that there is no magnetically disordered phase along the line $\lambda=1$.

Third, two mean field estimates of $\lambda^{c}\left(J_{y}\right)$ are presented in the figure. The dotted-dashed line is based on "dimer mean field theory," [16] that is, the zeroth- and first-order terms in $\chi(\lambda)$ are used to estimate $\lambda_{c}$. (This is equivalent to considering a single dimer subject to a staggered field which is determined, self-consistently, by its staggered magnetization.) That result is $\lambda_{\mathrm{DMF}}^{c}=1 /\left(1+2 J_{y}\right)$, and it has the remarkable (and accidental) feature that it yields the exact $\lambda^{c}$ when $J_{y}=0$. The broad solid line is based on the chain mean field theory discussed in Sec. 匹IB. The values of $\chi_{1}(\lambda)$ (the antiferromagnetic susceptibility for a single chain), which are the essential input into that mean field theory, were obtained by integrating a differential approximant to the seventh-order series presented in Ref. 14. The broad solid line ends at $J_{y} \approx 0.02$ because the numerical estimates of $\chi(\lambda)$ are not reliable for $\lambda$ arbitrarily close to 1 . Over most of the range of $J_{y}$ plotted, the dimer and chain mean field theories give quite close values of $\lambda^{c}$. The agreement is somewhat better than one would expect: in both cases the leading behavior of $\lambda^{c}$ at large $J_{y}$ is $1 / 2 J_{y}$, but the next-order terms are $-3 / 4 J_{y}^{2}$ and $-1 / 4 J_{y}^{2}$ for the chain and dimer mean field theories, respectively, and at $J_{y}=1$ the difference is hardly negligible! At small $J_{y}$ the chain mean field theory is clearly in better agreement with the series extrapolations than the dimer mean field theory. 
To briefly recapitulate, the dimer series for the two-dimensional lattice of coupled chains are entirely consistent with $J_{y}^{c}=0$. However, there is reason to doubt the strength of this conclusion. Since $\lambda^{c}(0)=1$ and $\lambda^{c}(1) \approx 0.54$, it would be entirely natural to conclude from too-short series that $\lambda^{c}\left(J_{y}\right)$ interpolates smoothly between these two endpoints, even if the correct result is that $\lambda^{c}$ does not exist for $0<J_{y}<J_{y}^{c}$ as would be the case if the scenario of Fig. 7(b) were to hold. As a partial test of the reliability of the series estimates of $\lambda^{c}\left(J_{y}\right)$, we have considered the problem of two coupled chains by the dimer series expansion method. The chains were taken to lie parallel to the bonds in the columnar dimer configuration. The only differences between these calculations and those preceding are the set of connected clusters (and their lattice constants), and that we will present results for $M_{x x}$ rather than $M_{r r}$. (In fact one can use $M_{x x}$ to estimate $\lambda^{c}\left(J_{y}\right)$ for the plane of chains as well; the differences between the estimates based on $M_{x x}$ and $M_{r r}$ are insignificant for the $J_{y}$ of interest.)

For two chains the only critical point in the $\lambda$ - $J_{y}$ plane is $\left(\lambda=1, J_{y}=0\right)$ [17]. Estimates of $\lambda^{c}\left(J_{y}\right)$ coming from inhomogeneous differential approximants to the $\chi$ and $M_{x x}$ series for two chains are displayed in Fig. 9, on the same scale as in Fig. 8 for ease of comparison. The thick solid and thin dotted-dashed curves are the chain and dimer mean field results, respectively, for two chains. (One obtains these from the mean field results for planes of chains by the substitution $2 J_{y} \rightarrow J_{y}$.) Several points are evident upon inspection. First, even at very small $J_{y}$ one can distinguish between the estimates of $\lambda^{c}$ from the differential approximants for the plane of chains and for two chains; they seem to approach zero with different slopes, on these plots. This could be taken as further evidence that, for the plane of chains, $J_{y}^{c}$ is extremely small if not vanishing.

However, for $J_{y} \lesssim 0.2$, where the approximants are well-converged, one might conclude that there are critical points at $\lambda \approx 1$, contrary to the known behavior of coupled pairs of chains. We believe this line segment of "pseudocritical" points reflects the existence of local maxima in the correlation length at $\lambda \approx 1$ for fixed, small $J_{y}$, where the correlation length at these maxima exceeds the typical cluster length in the seventh-order calculation 
(which is roughly 10). That such pseudocritical points should exist is entirely plausible: numerical studies for pairs of finite chains [4, 11] find that the gap initially decreases as $J_{y}$ is increased from zero. What is somewhat disturbing is that, for $J_{y} \lesssim 0.1$, the two-chain approximants are as consistent with chain mean field theory (for two chains) as the planeof-chain approximants are with chain mean field theory (for a plane of chains). Thus it is conceivable that for the plane of chains, the values of $\lambda^{c}$ indicated by the series at sufficiently small $J_{y}$ are pseudocritical points, rather than true critical points, as well.

To conclude, the problem of coupled chains at small $J_{y}$ poses significant challenges to numerical studies. Although the series expansions are consistent with $J_{y}^{c}=0$ they do not rule out a small but positive $J_{y}^{c}$. If the disordered phase exists, the correlation length along the $x$ direction is probably large throughout that phase, and the gap is everywhere small. A potentially fruitful avenue for future studies would be the consideration of models which include further-neighbor couplings; suitable models would have larger critical interchain couplings within spin-wave theory than the simplest coupled Heisenberg chain model studied here.

ACKNOWLEDGMENTS: IA thanks J. Fröhlich, M. Gingras, and E. Sørensen for helpful discussions. MPG thanks Keith Briggs, for sharing the code for his differential approximant calculation program "DA," and D. Frenkel for discussions. IA was supported in part by NSERC of Canada. MPG acknowledges support from the MacArthur Chair at the University of Illinois (and the hospitality of its holder, A. J. Leggett) during the initial stages of this work. RRPS was supported by NSF grant number DMR-9318537. 


\section{REFERENCES}

[1] P. W. Anderson, Phys. Rev. Lett. 64, 1839 (1990).

[2] T. Sakai and M. Takahashi, J. Phys. Soc. Japan 58, 3131 (1989).

[3] M. Azzouz, Phys. Rev. B 48, 6136 (1993).

[4] A. Parola, S. Sorella and Q. F. Zhong, Phys. Rev. Lett. 71, 4393 (1993).

[5] D. J. Scalapino, Y. Imry, and P. Pincus, Phys. Rev. B 11, 2042 (1975).

[6] S. T. Chui and M. R. Giri, Phys. Lett. A 128, 49 (1988); a summary of all numerical work to date is provided in Fig. 1 of B. Chattopadhyay and S. R. Shenoy, Phys. Rev. Lett. 72, 400 (1994).

[7] S. Hikami and T. Tsuneto, Prog. Theor. Phys. 63, 387 (1980).

[8] J. M. Kosterlitz and D. J. Thouless, in Progress in Low Temperature Physics Vol. 7B, edited by D. F. Brewer (North-Holland, Amsterdam, 1978).

[9] For a review, see I. Affleck, in Fields, Strings, and Critical Phenomena, edited by E. Brezin and J. Zinn-Justin (Elsevier, Amsterdam, 1990).

[10] F. D. M. Haldane, Phys. Rev. Lett. 61, 1029 (1988).

[11] T. Barnes, E. Dagotto, J. Riera, and E. S. Swanson, Phys. Rev. B 47, 3196 (1993).

[12] S. R. White, R. M. Noack, and D. J. Scalapino, preprint cond-mat/9403042.

[13] For analytical arguments see, for example, N. Read and S. Sachdev, Phys. Rev. Lett. 62, 1694 (1989); ibid. 66, 1773 (1991). For numerical studies which address the issue of nonmagnetic order in frustrated $S=1 / 2$ antiferromagnets see H. J. Schulz and T. A. L. Ziman, Europhys. Lett. 18, 355 (1992); E. Dagotto and A. Moreo, Phys. Rev. B 39, 4744 (1989), and Phys. Rev. Lett. 63, 2184 (1989); R. R. P. Singh and R. Narayanan, Phys. Rev. Lett. 65, 1072 (1990); M. P. Gelfand, Phys. Rev. B 42, 8206 (1990); M. P. 
Gelfand, R. R. P. Singh and D. A. Huse, Phys. Rev. B 4010801 (1989).

[14] M. P. Gelfand, R. R. P. Singh, and D. A. Huse, J. Stat. Phys. 59, 1093 (1990).

[15] M. E. Fisher and H. Au-Yang, J. Phys. A 12, 1677 (1979); D. L. Hunter and G. A. Baker, Jr., Phys. Rev. B 19, 3808 (1979).

[16] R. R. P. Singh, M. P. Gelfand, and D. A. Huse, Phys. Rev. Lett. 61, 2484 (1988).

[17] H. J. Schulz, Phys. Rev. B 34, 6372 (1986); S. P. Strong and A. J. Millis, Phys. Rev. Lett. 69, 2419 (1992). 


\section{FIGURES}

FIG. 1. The spatially anisotropic Heisenberg model. The solid and dashed segments correspond to interactions $J_{x}$ and $J_{y}$.

FIG. 2. Renormalization group flows for the half-integer spin quantum Heisenberg models. Here we assume $g_{2}(0)<g_{c}$ so the system orders for arbitrarily small $R$.

FIG. 3. Padé approximants for the ground state energy, evaluated at $\alpha=1$. The symbols are approximants to Ising series, while the lines (which are connecting points with $J_{y}=0,0.1,0.2$, 0.3, 0.4, 0.5, and 0.75) are approximants to dimer series.

FIG. 4. Padé approximants to the Ising expansion for the sublattice magnetization, evaluated at $\alpha=1$ following the change of variables described in the text.

FIG. 5. Padé approximants to the Ising expansion for the correlation length anisotropy, evaluated at $\alpha=1$.

FIG. 6. The columnar dimer covering $\mathcal{D}$ used for the dimer expansions; see Eq. (3.5).

FIG. 7. Sketches of the simplest plausible phase diagrams in the first quadrant of the $J_{y}-\lambda$ plane for the Hamiltonian (3.5) assuming that (a) $J_{y}^{c}=0$, and (b) $J_{y}^{c}>0$. Note that units of energy are chosen so that $J_{x}=1$. The phase diagrams must satisfy two contraints that follow from the invariance of correlation functions with respect to multiplication of the Hamiltonian by a constant: $\left(J_{y}, \lambda\right)=(a, 1)$ and $(1 / a, 1)$ lie in the same phase, and so must $(a, b)$ and $(a b, 1 / b)$. In both cases the hatched regions constitute the Néel ordered phase, which are surrounded by lines of critical points. The one-dimensional Heisenberg critical point at $\left(J_{y}, \lambda\right)=(0,1)$ is indicated by the large dot. The entire right boundaries of the plots, $J_{y}=\infty, 0<\lambda<\infty$, are also one-dimensional Heisenberg critical points. All other points are supposed to have only short-range correlations. 
FIG. 8. Estimated values of the critical $\lambda$ as a function of $J_{y}$ for the coupled-chain model (3.5). From the $\chi$ series, differential approximants displayed are $[2,4 ;-1](\bigcirc),[3,2 ; 0](\square)$, and $[3,3 ;-1](\diamond)$; from the $M_{r r}$ series, $[2,4 ;-1](\triangle),[3,3 ;-1](\nabla)$, and $[4,2 ;-1](\triangleright)$. See the text for a discussion of the significance of the various curves.

FIG. 9. The symbols correspond to estimates of the critical $\lambda$ for two chains, corresponding to the same approximants as the preceding figure (but for $M_{x x}$ rather than $M_{r r}$ ). The curves are discussed in the text. 


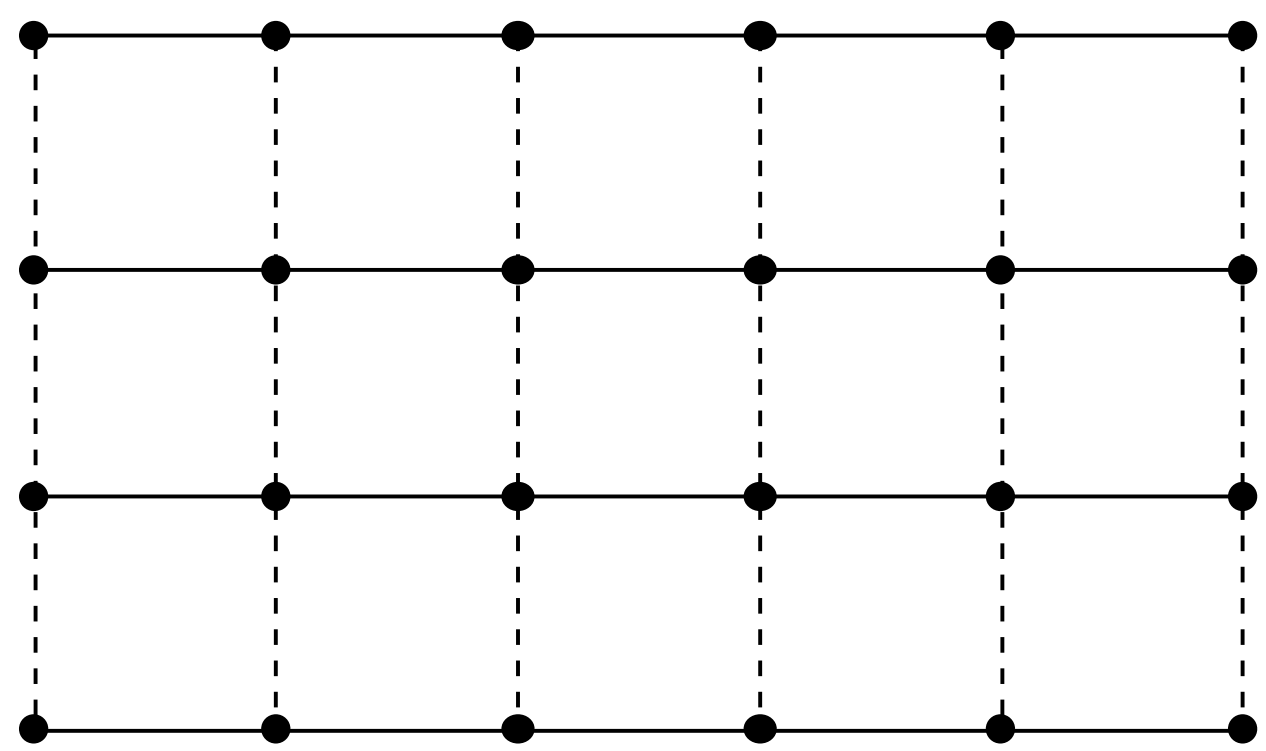




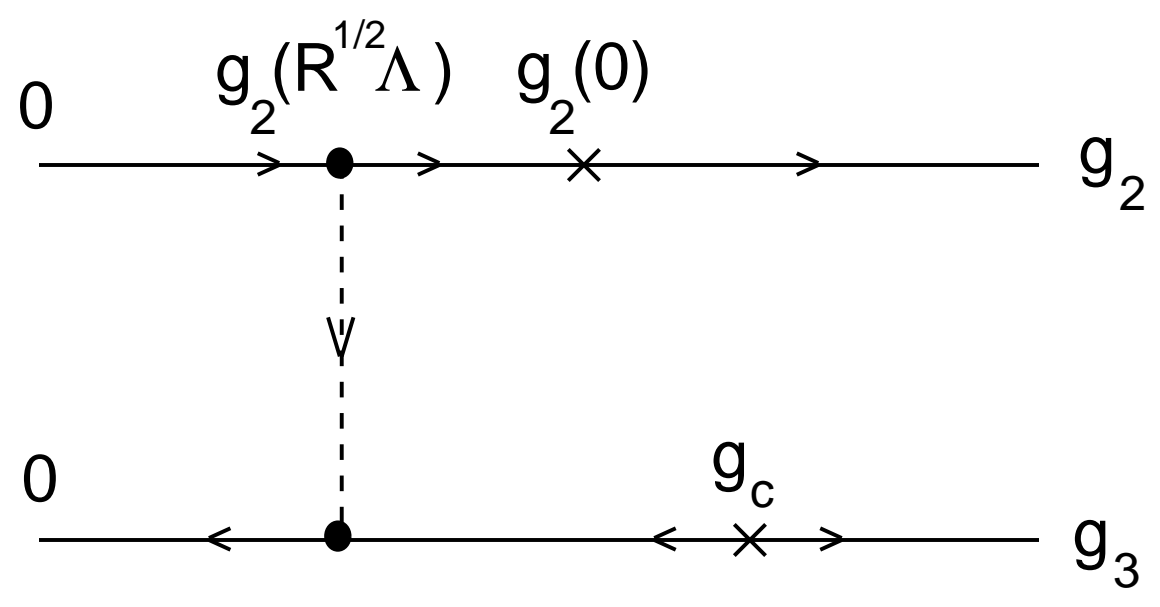




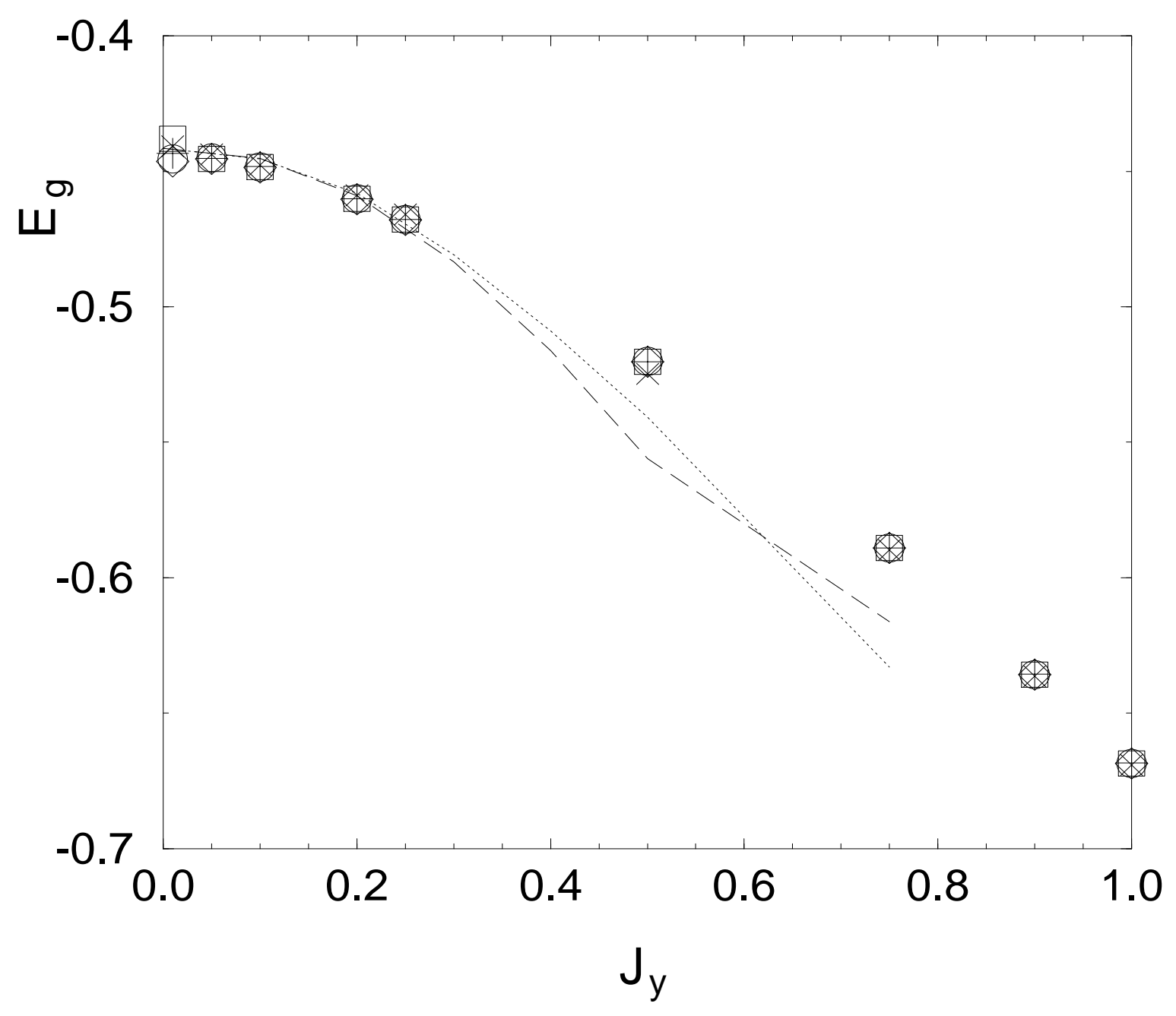




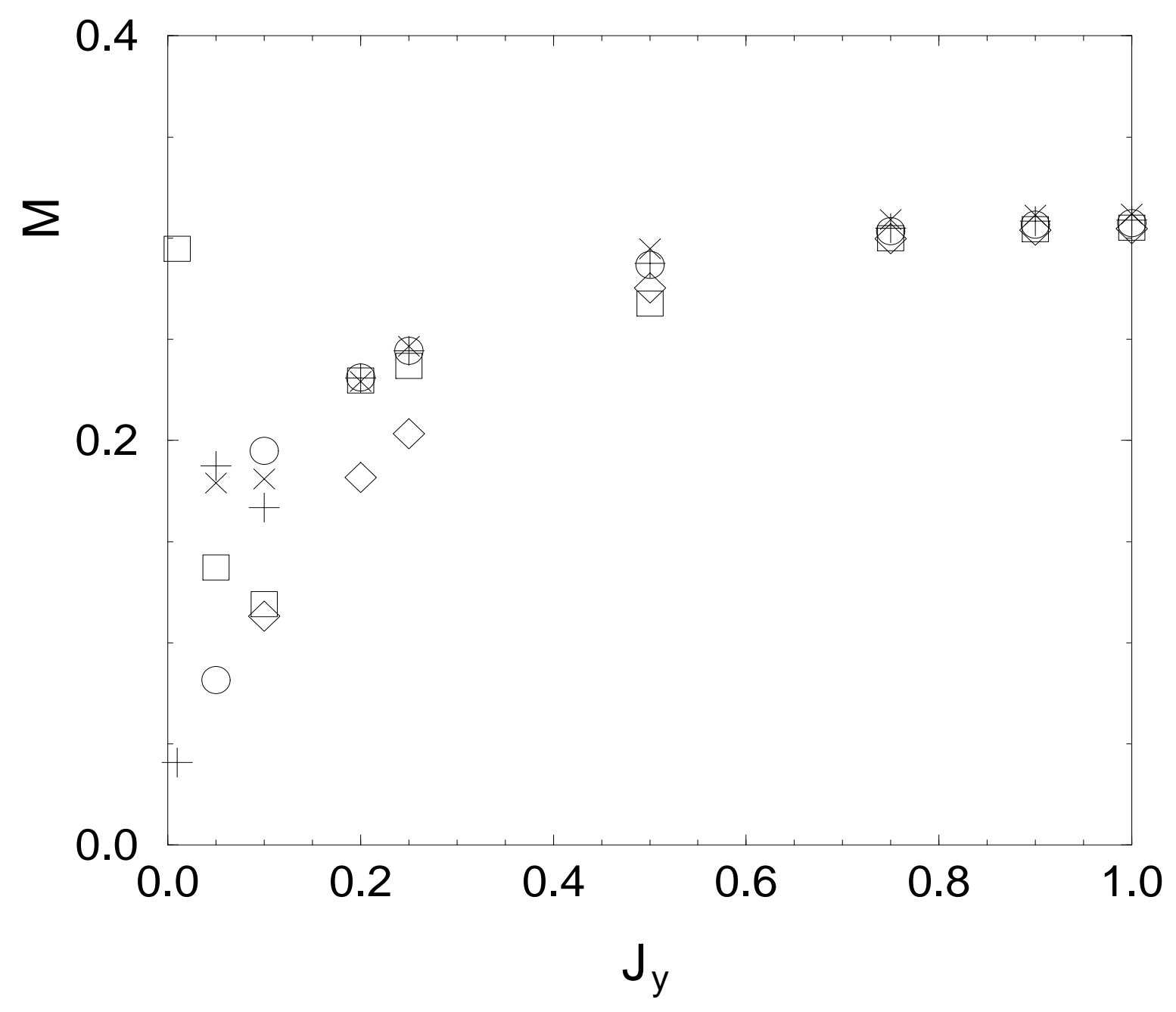




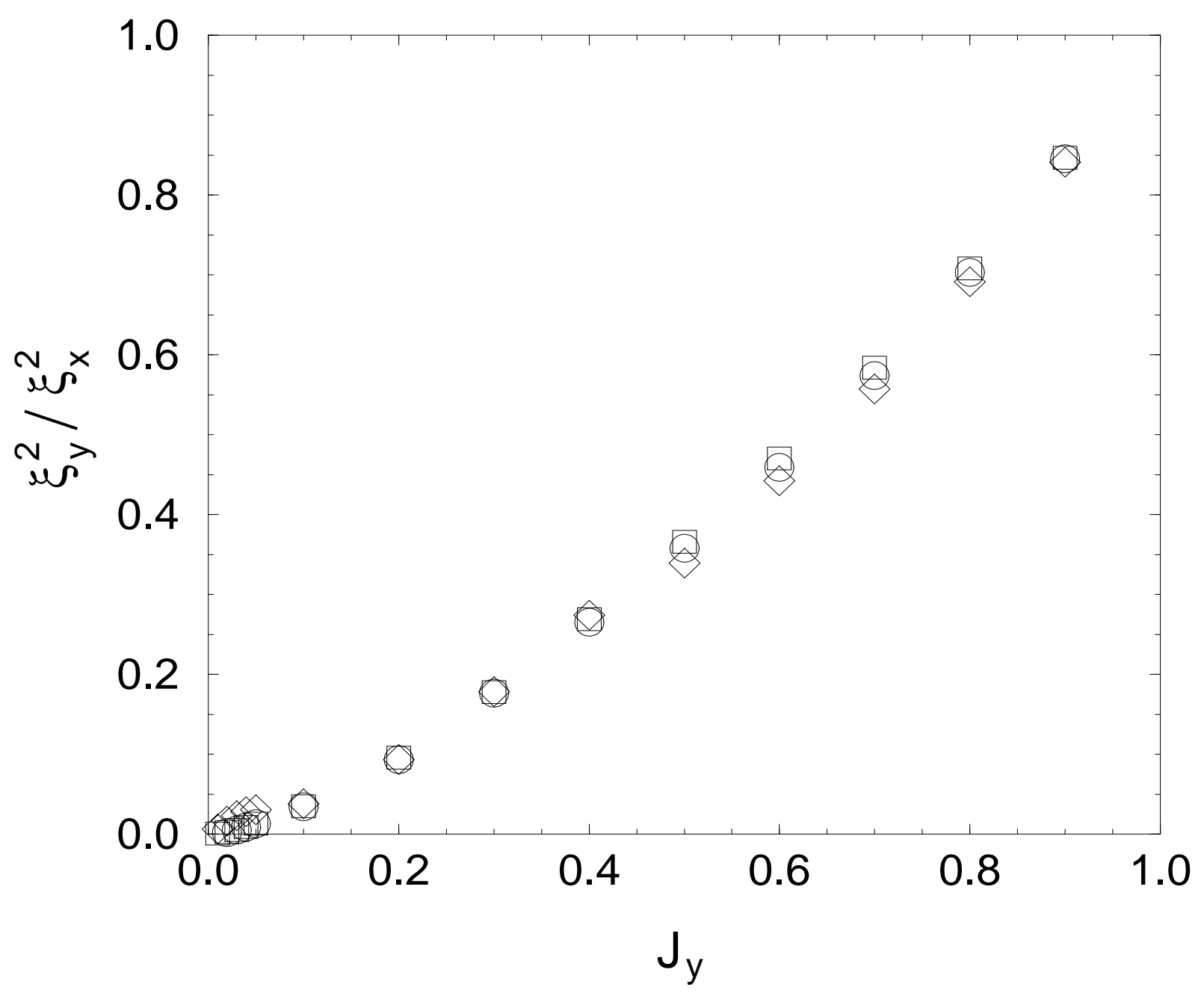




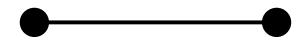

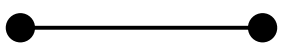

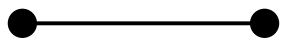

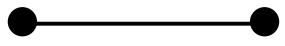

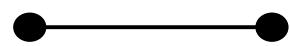

-

$\mathrm{O}$

-

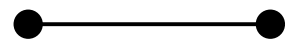

$\mathrm{O}$
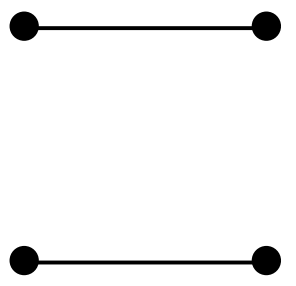


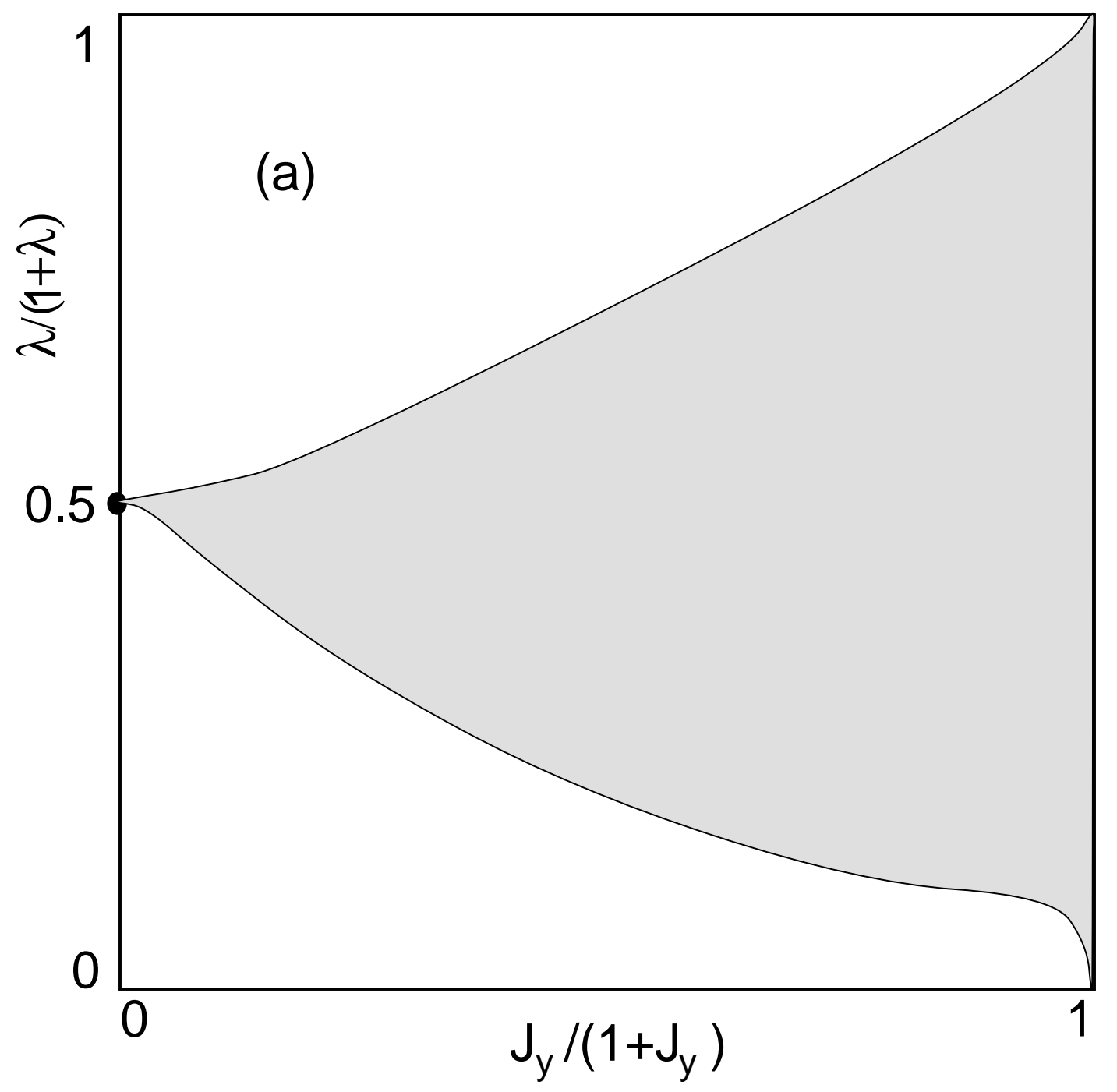




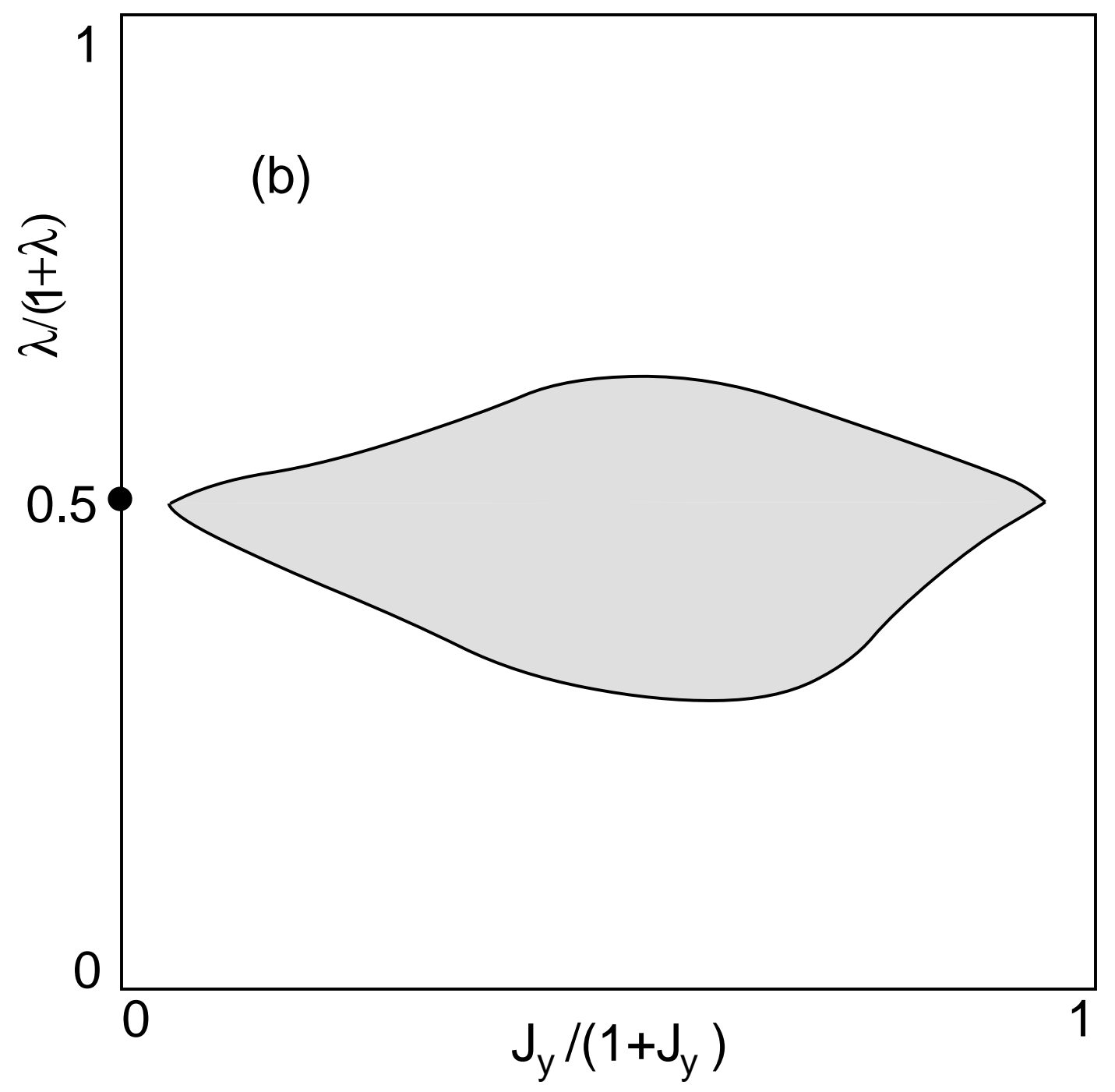




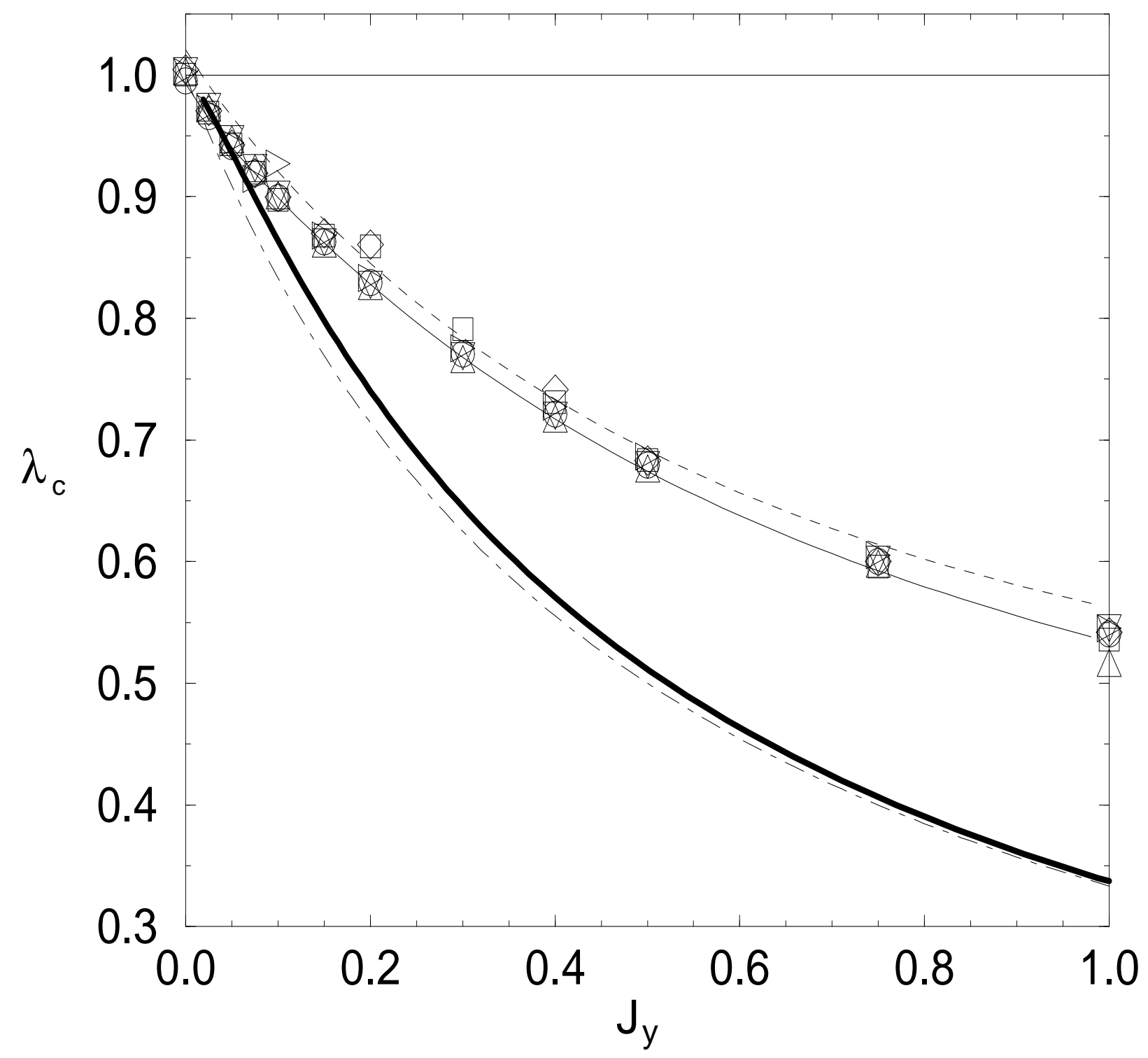




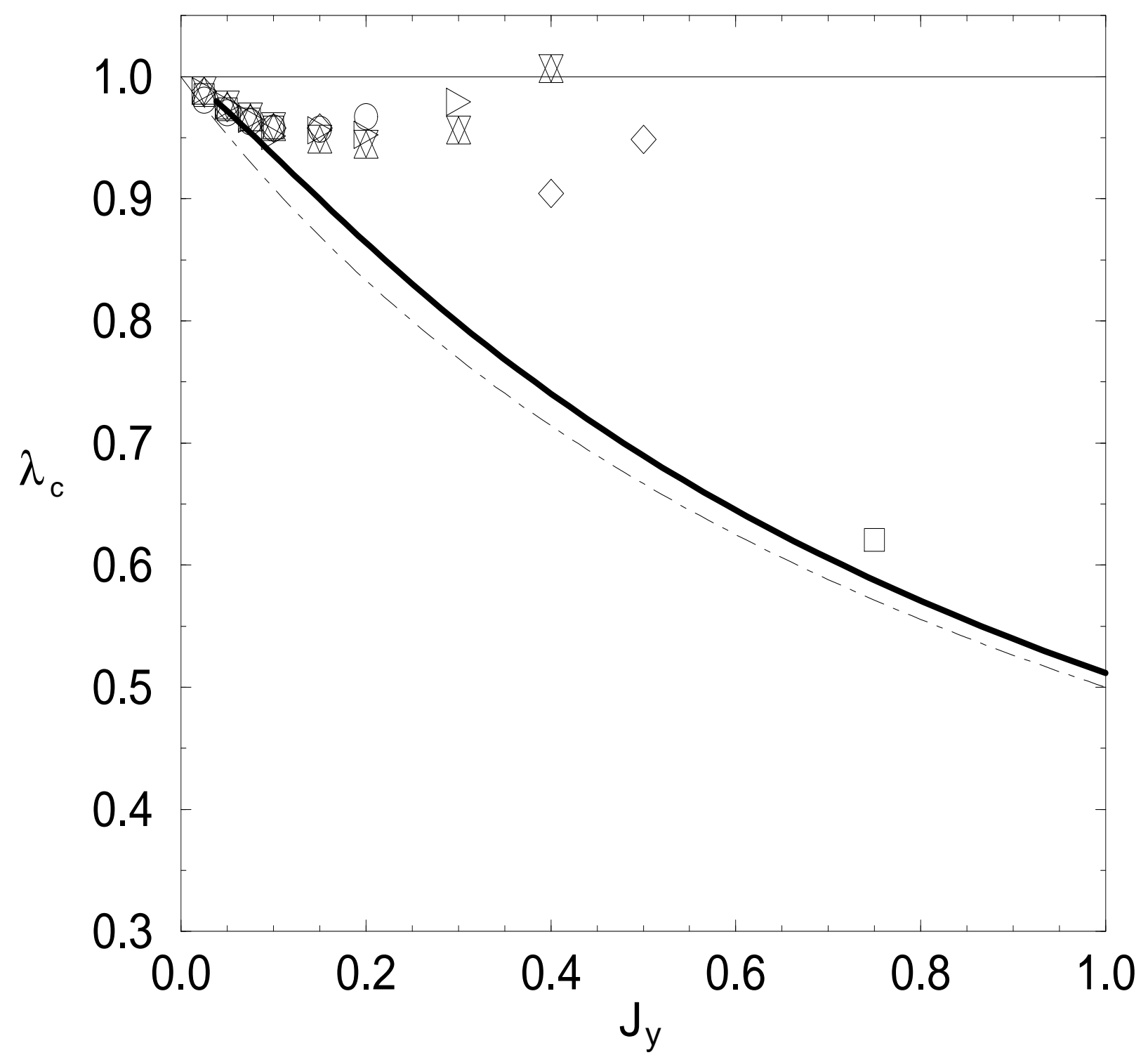

